\title{
ESTUDO DA ATITUDE E DO CONHECIMENTO DOS MÉDICOS NÃO ONCOLOGISTAS EM RELAÇÃO ÀS MEDIDAS DE PREVENÇ̧ÃO E RASTREAMENTO DO CÂNCER
}

\author{
luciana Tomanik Cardozo de Mello Tucunduva, Victor Hugo lara Cardoso de Sá, \\ Erika Tae Koshimura, fernanda Vilas Boas Prudente, Andréa felice dos Santos, \\ Eliana Sueco tibana Samano, Luciano José Megale Costa, Auro Del Giglio* \\ Trabalho realizado na disciplina de Oncologia e Hematologia da Faculdade \\ de Medicina do ABC, Santo André, SP.
}

RESUMO - OBjetivo. Avaliar o nível de informação e as atitudes preventivas em uso corrente pelos médicos ligados à FMABC.

Métodos. Foram entregues questionários para $I 20$ médicos não oncologistas que lidam diretamente com pacientes adultos.

Resultados. A taxa de resposta foi de $58,3 \%$. A idade média dos médicos foi de 33,9 anos, sendo $57,1 \%$ mulheres e $10 \%$ tabagistas. As práticas preventivas para os cânceres mais comuns (mama, colo de útero, próstata, colorretal e pele não-melanoma) foram analisadas e comparadas com as recomendadas pelos consensos adotados (INCA, Sociedade Americana de Cancerologia e Força-Tarefa Canadense). Observou-se que a maioria das práticas $(45,72 \%$ a $100 \%)$ não se adequou a nenhum deles. Sobre possíveis barreiras para o adequado exercício da prevenção do câncer, $82,86 \%$ considerou falta de agentes educadores em saúde para a população, $77,14 \%$ poucos conhecimento ou treinamento e $70,15 \%$ falta de verba para custear exames. Houve uma tendência ao excesso de pedidos de exames de rastreamento.

ConClusöes. As práticas preventivas utilizadas pelos médicos entrevistados são heterogêneas e, em sua maioria, não preconizadas pelos consensos de condutas preventivas consultados, o que pode ser relacionado tanto à sua falta de conhecimento em relação a estes consensos como às divergências entre eles. Dessa forma, faz-se necessário um esforço educativo enfatizando a importância da prevenção do câncer no aprendizado e na prática médica.

Unitermos: Prevenção primária. Câncer. Médicos. Consenso. Guia de prática médica.

\section{INTRODUÇÃO}

O câncer é a segunda causa de morte por doença no Brasil, precedido apenas pelas doenças cardiovasculares, exceto na região Nordeste do país, onde representa a terceira causa de morte por doença, precedido também pelas doenças infecciosas e parasitárias '. Dados do Instituto Nacional do Câncer (INCA), órgão de Saúde Pública do Ministério da Saúde responsável pela prevenção e controle do câncer, mostram que, em 1994, as neoplasias foram responsáveis por 10,86\% dos 887.594 óbitos registrados, sendo $53,81 \%$ entre homens e $46,05 \%$ entre as mulheres ${ }^{1,2}$.

A identificação dos estágios iniciais das doenças crônicas pode reduzir taxas de morbidade e mortalidade, o que pode ser realizado por meio de três níveis de programas de prevenção: a primária previne a ocorrência da enfermidade, a secundária consiste no diagnóstico precoce por meio de rastreamento e a

\section{* Correspondência:}

Avenida Rebouças, 3387 - CEP:05410-040

São Paulo - S P - Brasil - Tel/Fax: (11) 3819-5007 sandrabr@netpoint.com.br terciária previne deformidades, recidivas e morte $^{3}$

No caso do câncer, a prevenção primária consiste na limitação da exposição a agentes causais ou fatores de risco (relacionados a 80\% dos tumores) como fumo, asbesto, sedentarismo, dieta inadequada, vírus (papiloma vírus e vírus da hepatite B) e exposiç̧ão solar. A prevenção secundária do câncer requer procedimentos junto à população que permitam o diagnóstico precoce ou detecção das lesões pré-cancerosas, cujo tratamento pode levar à cura ou, ao menos, à melhora da sobrevida dos indivíduos. Com os conhecimentos atuais, sabe-se que a prevenção primária somada à secundária, ou seja, diminuição da exposição da população a fatores de risco e diagnóstico precoce pode reduzir em 2/3 o número de casos de câncer ${ }^{3}$. Outros estudos mostram, ainda, que a prevenção efetivamente reduz a mortalidade por câncer em geral, é capaz de diminuir tanto a incidência como a mortalidade relacionadas ao câncer de colo de útero e reduzir até 29\% das mortes por câncer de mama ${ }^{4,5,6}$. Portanto, é necessária uma constante atualização de todos os profissionais da saúde em relação aos programas de prevenção e, no câncer em especial, quanto às medidas primárias e secundárias ${ }^{7}$

As estratégias de prevenção das doenças crônicas exigem um forte embasamento científico. Somente a partir da década de 60 houve um incremento nos estudos de efetividade das estratégias preventivas, levando à implementação de programas preventivos sistemáticos ${ }^{2}$.

Atualmente, não há grandes estudos populacionais de prevenção do câncer na população brasileira, de forma que as medidas recomendadas pelo INCA são baseadas em estudos realizados por entidades interna cionais, particularmente americanas (American Cancer Society - ACS) e canadenses (Canadian Task Force - CTF). Estas medidas foram publicadas após um consenso entre autoridades no assunto que avaliaram o nível de evidência e o grau de recomendação para cada tipo de câncerer, $2,8,9$.

Ashford e colaboradores estudaram o conhecimento de médicos de uma cidade do interior dos Estados Unidos sobre as recomendações da ACS e constataram que $70 \%$ dos médicos afirmam ter conhecimento destas 
medidas. Entretanto, $77 \%$ consideram o treinamento do médico para fazer prevenção insuficiente e $43 \%$ afirmam que as recomendações não são claras, de maneira que 17\% responderam incorretamente sobre as medidas de prevenção do câncer colorretal, 53\% acreditam que a prevenção de câncer de pulmão seja realizada com radiografia de tórax para todos os pacientes tabagistas, $68 \%$ acreditam que as recomendações para câncer de próstata consistam apenas na dosagem de antígeno prostático específico (PSA), entre outras medidas que não são as preconizadas pela ACS, o que sugere que o conhecimento dos médicos sobre a prevenção e rastreamento do câncer seja insuficiente ${ }^{10}$.

Não há estudos na literatura nacional que abordem o conhecimento dos médicos não oncologistas acerca das medidas de prevenção do câncer, apesar de serem estes profissionais os responsáveis pela maioria dos diagnósticos de câncer e, portanto, desempenharem um papel fundamental na prevenção e na detecção precoce desta doença ${ }^{\prime \prime}$.

Os objetivos deste trabalho são avaliar o conhecimento e a atitude dos médicos não oncologistas em relação às medidas de prevenção do câncer, tendo como base os consensos do INCA, ACS e CTF, assim como conhecer os principais fatores que possam afetar o adequado exercício da prevenção do câncer pelos médicos não oncologistas em nosso meio.

\section{Métodos}

O estudo foi realizado por meio de um questionário intitulado "Médicos e Prevenção do câncer", que foi aplicado, após aprovação pelo Comitê de Ética em Pesquisa da Faculdade de Medicina do ABC, a I 20 médicos não oncologistas vinculados a esta instituição, durante o mês de maio de 2003. Com o intuito de atingir os objetivos da pesquisa, a amostra foi composta por médicos não oncologistas que estabelecessem contato direto com pacientes adultos.

O questionário foi composto por três partes: identificação do médico por meio de dados pessoais como especialidade, ano de graduação, idade, sexo, local de conclusão do curso e hábito tabagista; 14 questões sobre atitude e conhecimento do médico em relação à prevenção das neoplasias malignas mais

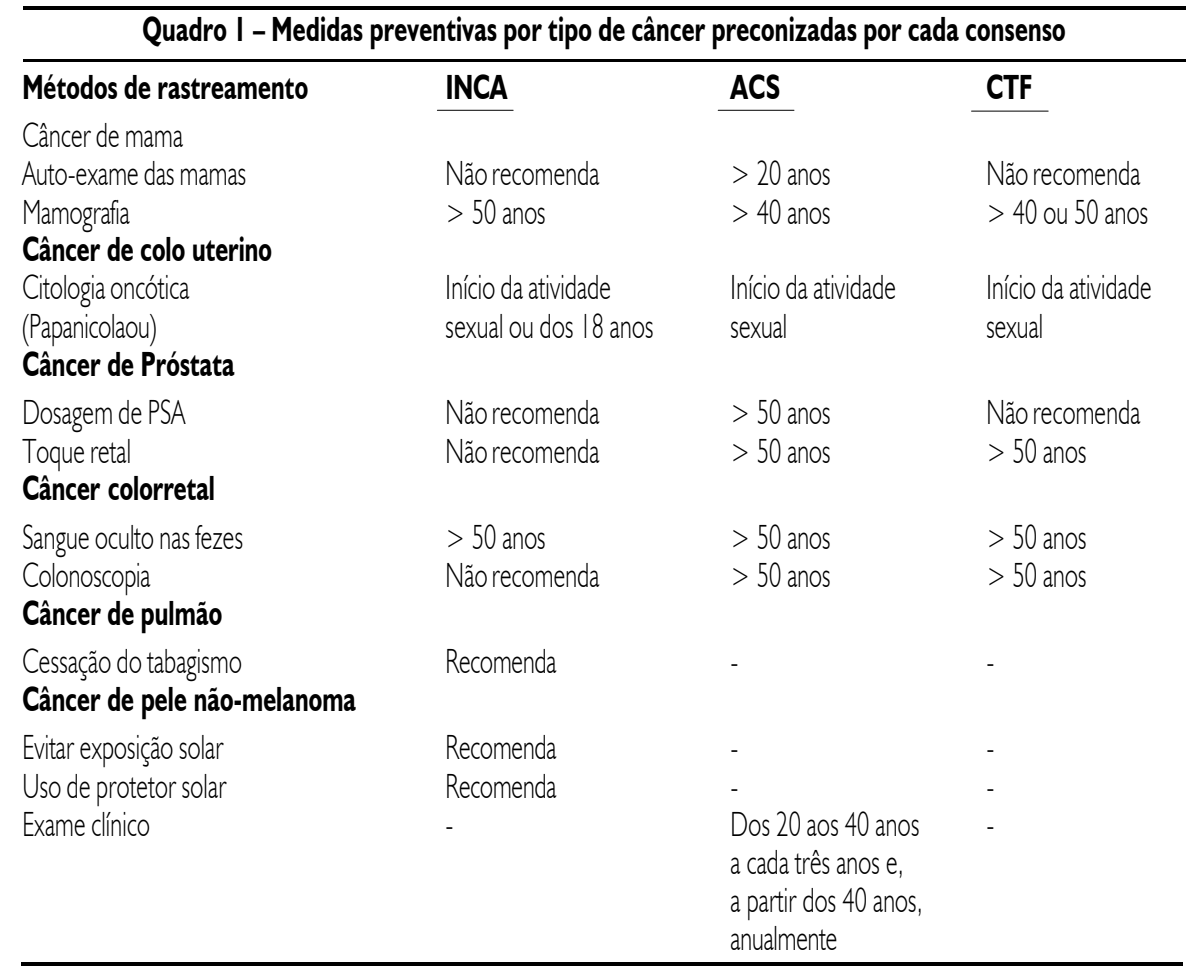

INCA, ACS, CTF

prevalentes no Brasil, ou seja, câncer de mama, próstata, pulmão, pele não-mela noma, colo de útero e colorretal, e graduação da importância de itens considerados como barreiras ao adequado exercício da prevenção do câncer. A análise das respostas foi baseada nos consensos do INCA, da ACS e da CTF (Quadro I).

Para a estatística descritiva utilizamos o programa Windows Excel do Windows Millenium Edition ME. A análise estatística foi realizada utilizando-se o programa NCSS 2000PASS 2000 (Utah, EUA). Para as variáveis discretas foi aplicado o teste do qui-quadrado e para comparação das médias das variáveis contínuas foi usado o teste paramétrico t de Student. O teste de regressão foi aplicado para avaliar o relacionamento entre diferentes variáveis. Foi considerado com significância estatística o valor de $p<0,05$.

Consideramos conduta adequada a indicação de exames de prevenção que estivessem de acordo com pelo menos um consenso de conduta consultado. As condutas inadequadas, por sua vez, foram consideradas aquelas que não se enquadravam em nenhum dos consensos utilizados e foram subdivididas em: excessiva, se fosse indicada uma atitude preventiva para um paciente que não a necessitasse, e deficiente, se não fosse indicada uma conduta preventiva para um paciente que a necessitasse.

\section{Resultados}

A taxa de resposta foi de $58,30 \%$ ou 70 médicos, com média de idade de 33,9 anos (desvio padrão de 10,4 anos) e, em média, 8,5 anos de formados (desvio padrão de 9,6 anos). Quanto às características demográficas, 67,10\% dos médicos tinham formação clínica, com distribuição por especialidades descritas na tabela I, 57, 10\% eram do sexo feminino e $90 \%$ não tabagistas.

Dos entrevistados, 67,10\% questionam suas pacientes se elas fazem exames de rastreamento do câncer de mama, mas apenas 24,30\% ensinam suas pacientes a fazer o autoexame das mamas durante a consulta. Com relação ao tabagismo, 97, 10\% incentivam seus pacientes a cessarem o tabagismo, sendo somente $\mathrm{o}$ aconselhamento $(55,71 \%)$ a forma mais utilizada de incentivo. Quando questionam seus pacientes se eles se submetem a exames de rastreamento, os médicos dão maior ênfase aos cânceres de colo de útero e mama, como descrito na Tabela 2. 
Em relação às medidas de rastreamento dos tipos de câncer analisados, foi observado que, em sua maioria, não houve correlação entre as respostas dos médicos e os consensos, com taxa de erro das respostas variando entre $45,72 \%$ e $100 \%$. Os tipos de câncer cujas respostas revelaram menor conformidade com os consensos adotados foram mama e colorretal. Os cânceres de pulmão e pele nãomelanoma demonstraram os maiores índices de conformidade com os consensos, $54,28 \%$ e $51,42 \%$, respectivamente (Tabela 3).

Na Tabela 4, analisamos a variação das condutas de rastreamento em relação aos consensos da ACS, CTF e do INCA praticadas pelos médicos entrevistados, diferenciando-as em condutas excessivas ou deficientes. A tabela demonstra que a indicação inadequada de todos os exames avaliados tendeu ao excesso.

A medida preventiva mais indicada para o câncer de pele não-melanoma foi a diminuiç̧ão da exposição solar somada ao uso diário de protetor solar (51,42\%). Outras medidas referidas pelos médicos foram exames clínicos profiláticos e dermatoscopia de lesões suspeitas, uso de barreiras mecânicas à exposição solar, não utilização de métodos de bronzeamento artificial e orientação quanto às alterações das lesões suspeitas.

Quando interrogados quanto a possíveis barreiras para o adequado exercício do rastreamento do câncer (Tabela 5), os médicos consideraram falta de agentes educadores em saúde para a população $(82,86 \%)$ e sua própria falta de conhecimento ou treinamento para fazer este tipo de rastreamento $(77,14 \%)$ de grande importância.

Quanto às correlações estatísticas, foi observado que os médicos com formação clínica tenderam a tomar conduta deficiente relacionada ao auto-exame das mamas de acordo com o preconizado pela ACS $(p=0,044)$, não ensinar o auto-exame das mamas $(p=0,00 \mathrm{I}) \mathrm{e}$ tomar conduta deficiente em relação ao PSA, de acordo com a ACS $(p<0,00 I)$.

Houve também correlação estatisticamente significante entre ser ginecologista e obstetra e questionar se suas pacientes realizam exames preventivos relacionados ao câncer de mama $(p=0,0 \mid 6)$, ensinar 0 autoexame das mamas $(p<0,001)$ e tomar conduta excessiva em relação ao Papanicolaou, de acordo com a ACS e CTF $(p=0,029)$. Da mesma forma, as médicas tenderam a tomar

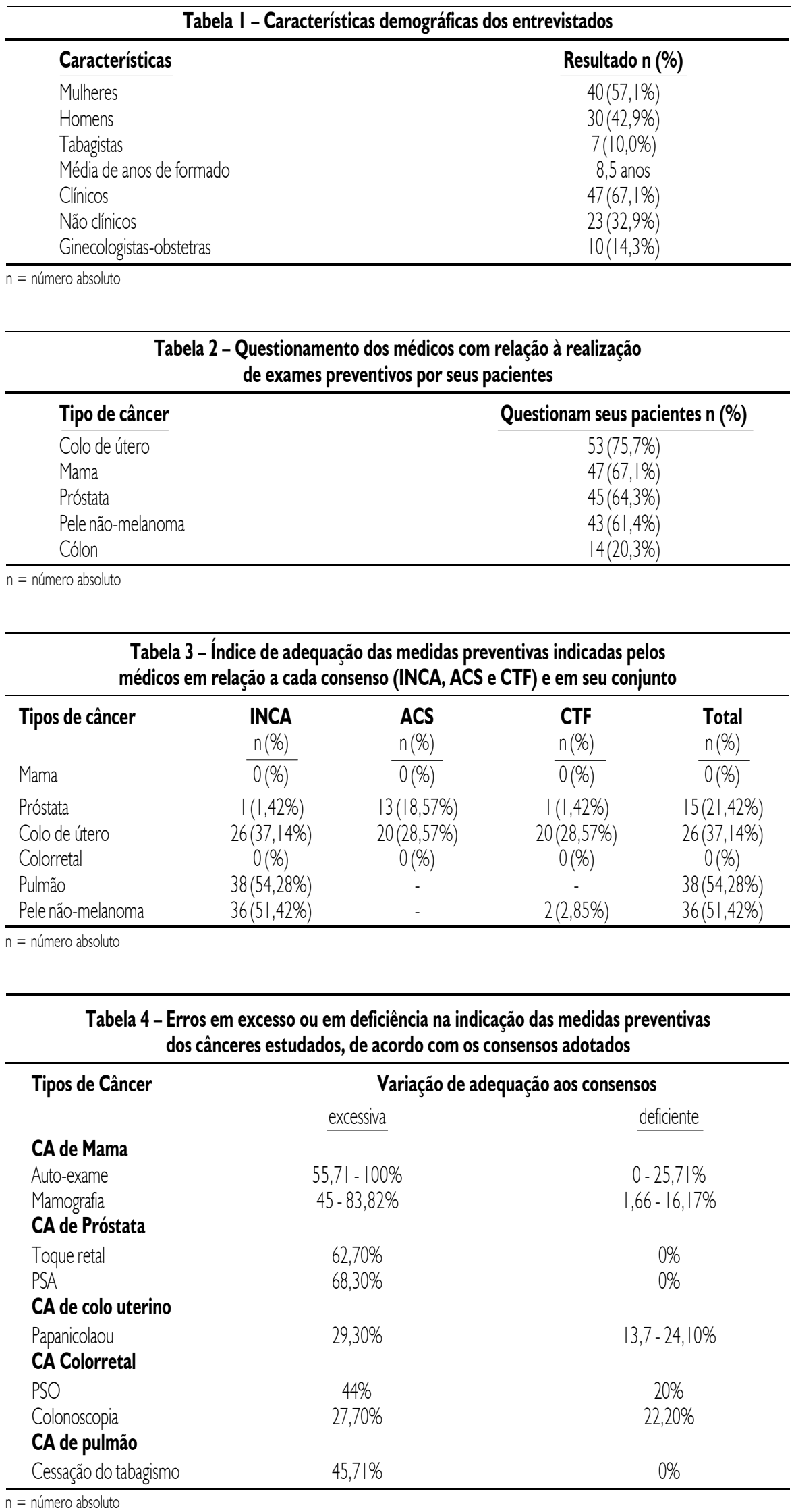


Tabela 5 - Indicação do grau de importância das bareiras que impedem 0 adequado exercício da prevenção do câncer

\begin{tabular}{|c|c|c|c|}
\hline Barreira & $\begin{array}{c}\text { Grande } \\
\text { importância } \\
n(\%)\end{array}$ & $\begin{array}{c}\text { Pouca } \\
\text { importância } \\
n(\%)\end{array}$ & $\begin{array}{c}\text { Não considero } \\
\text { uma barreira } \\
n(\%)\end{array}$ \\
\hline $\begin{array}{l}\text { Falta de agentes educadores em } \\
\text { saúde para a população como um todo } \\
\text { (agentes de saúde, enfermeiros, } \\
\text { nutricionistas e outros) }\end{array}$ & $\overline{58(82,86 \%)}$ & $9(12,86 \%)$ & $3(4,29 \%)$ \\
\hline $\begin{array}{l}\text { Falta de tempo do médico para } \\
\text { conversar com seus pacientes } \\
\text { sobre estes assuntos }\end{array}$ & $50(7 \mid, 43 \%)$ & $7(10,00 \%)$ & $13(\mid 8,57 \%)$ \\
\hline $\begin{array}{l}\text { O tempo entre uma consulta e a } \\
\text { próxima, do mesmo paciente, ser } \\
\text { espaçada, dificultando um seguimento } \\
\text { adequado. }\end{array}$ & $28(40,00 \%)$ & $30(42,86 \%)$ & $12(17,14 \%)$ \\
\hline $\begin{array}{l}\text { Falta de interesse do paciente } \\
\text { Falta de verba para custear }\end{array}$ & $42(60,87 \%)$ & $\begin{array}{c}1 \\
2(17,39 \%)\end{array}$ & $15(21,74 \%)$ \\
\hline $\begin{array}{l}\text { os exames de prevenção } \\
\text { As recomendações para se fazer } \\
\text { prevenção destas doenças não } \\
\text { são muito claras }\end{array}$ & $\begin{array}{l}47(70,15 \%) \\
31(45,59 \%)\end{array}$ & $\begin{array}{l}11(16,42 \%) \\
20(29,41 \%)\end{array}$ & $\begin{array}{l}9(13,43 \%) \\
17(25,00 \%)\end{array}$ \\
\hline $\begin{array}{l}\text { Os médicos têm pouco conhecimento } \\
\text { e/ou treinamento inadequado para } \\
\text { fazer este tipo de prevenção }\end{array}$ & $54(77,14 \%)$ & $8(11,43 \%)$ & $8(11,43 \%)$ \\
\hline
\end{tabular}

medidas excessivas em relação ao auto-exame das mamas de acordo com o INCA $(p=0,027)$ e a questionar se suas pacientes se submetem a exames de rastreamento do câncer de colo uterino $(p=0,036)$.

Médicos com mais de dez anos de formados tenderam a tomar conduta deficiente em relação ao auto-exame das mamas $(p=0,034)$ $e$, de acordo com o INCA, a não ensinar o auto-exame das mamas $(p=0,009)$ e a tomar conduta deficiente em relação ao Papanicolaou, de acordo com o INCA $(p=0,0 \mid 6)$, a ACS $(p=0,036)$ e a CTF $(p=0,036)$.

Médicos com menos de dez anos de formados questionam menos se seus pacientes fazem exames para rastreamento do câncer de próstata $(p=0,038)$ e tomam conduta inadequada no rastreamento do câncer colorretal em relação aos três consensos $(p=0,002)$.

\section{Discussão}

Os tipos de câncer com os quais os médicos demonstraram maior preocupação em relação às medidas de rastreamento foram colo de útero $(75,70 \%)$ e mama $(67,10 \%)$, com uma taxa muito baixa para o câncer colorretal (20,30\%), confirmando dados encontrados por Garcia-Gianolli e Sasco $(1996)^{12}$.

De uma maneira geral, os médicos demonstraram pouco conhecimento sobre as medidas preconizadas pelos consensos adotados, já que as taxas de respostas em conformidade com pelo menos um dos consensos foram muito baixas e, em sua maioria, correspondendo a menos de 50\%. As taxas de conformidade com os consensos maiores de $50 \%$ se referem aos cânceres de pulmão e pele não-melanoma e representam as respostas de apenas um pouco mais da metade dos médicos (Tabela 3).

Em relação ao câncer de mama, notamos adotados, já que nenhum médico apresentou respostas em conformidade com algum dos três consensos e que apenas a minoria $(24,3 \%)$ deles ensina regularmente 0 auto-exame das mamas, taxa muito menor que a encontrada em outros estudos, que varia entre $85 \%$ e $92 \%{ }^{12,16}$. O auto-exame não é preconizado de forma isolada pelo INCA e pelo CTF, que o inadequação das medidas preventivas indicadas pelos médicos em relação aos consensos indicam apenas para as mulheres que fazem exame clínico e mamografia regularmente, já que não existem dados que comprovem o benefício desta forma de prevenção (redução da mortalidade) e há evidências de prejuízo, como o aumento do número de biópsias de lesões benignas, falsa sensação de segurança nos falsos negativos e impacto psicológico negativo nos falsos positivos 9,2 . Por outro lado, o auto-exame é preconizado por outros consensos, como o da ACS, e citado como medida eficaz de rastreamento de câncer de mama ${ }^{8,14,17}$. A controvérsia existente em relação à eficácia do auto-exame, entretanto, não deve ser extrapolada para o exame físico periódico das mamas executado por um médico para o qual existem evidências na literatura indicativas de benefício ${ }^{18}$.

Apesar de a maioria dos médicos indicar a mamografia como método preventivo, a maior parte deles o faz para pacientes mais jovens do que o preconizado, o que condiz com o encontrado em outros estudos $10,16,12,4$.

Há controvérsias na literatura quanto à eficácia da mamografia como prática de rastreamento em mulheres entre 40 e 49 anos, apesar de ser indicada para pacientes maiores de 40 anos em alguns consensos, como o da ACS 9,4,19,8. Entretanto, a taxa de recomendação da mamografia para mulheres entre 40 e 49 anos vem se tornando semelhante à da faixa etária entre 50 e 59 anos ${ }^{19}$. Mesmo sendo onerosa para o sistema de saúde, a indicação excessiva de mamografia provavelmente é bem menos prejudicial para as pacientes do que a falta de indicação, já que foi constatado que o maior motivo pelo qual as pacientes não fazem exames de rastreamento do câncer de mama é a falta de indicação médica, comprovando a importância dos médicos generalistas na prevenção do câncer ${ }^{14,15}$.

Não há consenso em relação aos métodos preventivos para câncer de próstata. Enquanto urologistas preconizam a dosagem de PSA sérico e o toque retal anuais para homens maiores de 50 anos, os consensos de prevenção do câncer são heterogêneos, indicando PSA somado ao toque retal, apenas o toque ou nenhum deles (Quadro I) 8,9,2. A diversidade das respostas é confirmada pela literatura, com indicações de avaliação do PSA variando entre $68 \%$ e $73 \%$ em dois diferentes estudos. A dosagem do PSA sérico vem sendo cada vez mais utilizada pelos médicos ${ }^{19}$. 
No que diz respeito ao câncer de pulmão, a única medida preventiva comprovadamente eficaz é a cessação do tabagismo, que foi indicada por praticamente todos os médicos, de forma semelhante ao encontrado por Battista et al. ${ }^{16}$. O aconselhamento sem a utilização de medicamentos foi a maneira de incentivo à cessação do tabagismo mais utilizada, a despeito das evidências quanto à eficácia do uso de medicação no auxílio do abandono do tabagismo ${ }^{17}$. No entanto, a indicação de radiografia de tórax, que não é preconizada por nenhum consenso para rastreamento deste tipo de câncer, foi citada por quase metade $(45,7 \%)$ dos médicos, com taxas maiores encontradas na literatura, variando de $53 \%$ a $77 \%$ 10,16.

Em outros estudos referentes ao câncer de colo de útero foi encontrada maior taxa de indicação do exame de Papanicolaou em comparação aos nossos resultados, porém, todos eles apontam baixas taxas de indicação adequada de acordo com os consensos de prevenção ${ }^{10,16,12,2}$. A maior parte das indicações inadequadas tendeu ao excesso, o que condiz com o encontrado por outros autores. Contudo, a minoria que indica o exame de Papanicolaou de forma deficiente é significativa e deve ser conscientizada da importância deste método preventivo ${ }^{13,20}$.

As questões sobre as medidas preventivas em relação ao câncer colorretal obtiveram o menor índice de respostas e de adequação, já que nenhum médico indicou os exames preventivos de acordo com as condutas descritas nos consensos adotados, resultado mais desfavorável que o encontrado por outro autores ${ }^{10,1}{ }^{2}$. Destacamos também que, em nosso meio, a falta de adesão dos médicos também se refletiu na baixa adesão à recomendação para que se procedesse à pesquisa de sangue oculto nas fezes, que é uma medida barata e simples cuja eficácia foi já foi amplamente comprovada na literatura ${ }^{21}$.

As recomendações preventivas para 0 câncer de pele divergem nos consensos adotados. O INCA recomenda a diminuição da exposição solar, o uso diário de protetor solar e o exame clínico da pele, este último como modalidade preferida de rastreamento, apesar de não demonstrar redução da mortalidade ${ }^{2}$. Neste estudo, a conduta preventiva mais adotada foi o uso de protetor solar diário somado à diminuição da exposição ao sol. No estudo de Garcia-Gianolli e Sasco, 78\% dos médicos referiram fazer rastreamento deste tipo de câncer pela inspeção visual' ${ }^{12}$.

As barreiras referidas como de grande importância para o adequado exercício da prevenção do câncer foram: falta de agentes educadores em saúde, falta de conhecimento e treinamento dos médicos, falta de tempo para aconselhamento de seus pacientes e deficiência de recursos. As maiores semeIhanças com o estudo de Ashford e colaboradores foram a falta de prática na prevenção do câncer e de homogeneidade das recomendações de prevenção presentes na literatura. De forma diferente, em nosso estudo, os médicos entrevistados consideraram importantes a falta de agentes educadores em saúde e de recursos deficientes, o que não foi citado como tendo grande importância pela maioria dos médicos entre vistados no estudo de Ashford ${ }^{10}$. Esses resultados indicam que os médicos reconhecem sua falta de conhecimento sobre as medidas corretas para prevenção do câncer e sentem dificuldade frente à falta de uniformidade dos consensos para as mesmas.

Concluímos que os médicos entrevistados neste estudo demonstraram interesse em prevenir os diferentes tipos de câncer, mas apresentam dificuldade em fazê-lo da maneira preconizada pelos consensos de prevenção, tanto pela divergência entre estes como pela sua falta de conhecimento em relação aos consensos existentes. Dessa forma, para aumentar a adesão dos médicos aos consensos, são necessários recursos e esforços educativos. A partir de nossas conclusões, entendemos ser necessário um esforço multidisciplinar enfatizando a importância das medidas preventivas no aprendizado médico.

\section{Conflito de interesse: não há.}

\section{SUMMARY}

\begin{tabular}{llrr}
\multicolumn{2}{c}{ EVALUATION OF } & \multicolumn{2}{c}{ NON-ONCOLOGIST } \\
PHYSICIAN'S & KNOWLEDGE & AND ATTITUDE \\
TOWARDS & CANCER & SCREENING & AND \\
PREVENTIVE & ACTIONS & &
\end{tabular}

BACKGOUND. New cancer cases are most often diagnosed by non-oncologist phy sicians. It is therefore essential for all physicians to be aware of cancer preventive practices and use them appropriately with their patients.
Methods. Questionnaires were administered to 120 non-oncologist physicians of various specialties attending the "Faculdade de Medicina do ABC" who deal directly with adult patients. Replies were collected and classified as appropriate or not according to one of these three cancer prevention guidelines: INCA, American Cancer Society and Canadian Task Force.

RESULTS. The percentage of replied questionnaires was $58.3 \%$ (70 questionnaires). Mean age of physicians was 33.9 years; $57.1 \%$ were women and $10 \%$ smokers. Most of the current preventive practices adopted by the participating physicians (45.72\% to 100\%) regarding the most common and preventable tumors (breast, cervix, prostate, colon and rectum and non-melanoma skin cancer) did not agree with any of the guidelines mentioned above. When questioned about possible impediments for the appropriate practice of cancer prevention, $82.86 \%$ reported absence of health education agents working with the population, $77.14 \%$ scarceness of knowledge or training concerning prevention, and $70.15 \%$ lack of financial supportfor ordering tests. Frequently, whenever there was disagreement between the guidelines and the physician's current practices, preventive tests were ordered in excess of those recommended by the guidelines.

Conclusions. Physicians had a tendency to order excessive laboratory tests, an action which can be related to lack of knowledge and to divergence among guidelines. A more intensive educational effort regarding cancer prevention, directed towards teaching physicians in training, seems to be warranted. [Rev Assoc Med Bras 2004; 50(3): 257-62]

KEY wORDS: Primary prevention. Cancer. Physicians. Consensus. Practice guidelines.

\section{RefERÊNCIAS}

I. Ministério da Saúde. Instituto Nacional do Câncer. Coordenação de programas de controle de câncer - Pro-Onco. "O câncer no Brasil". [online]. Disponível em: http:// www.inca.org.br/cancer/epidemiologia/ cancer_no_brasil.html. Acesso em: jan 2003.

2. Normas e recomendações do INCA. Prevenção e controle do câncer. Rev Bras Cancerologia 2002; 48:317-33.

3. Antunes RCP. Recomendações atuais na prevenção do câncer no Brasil. Prática Hospitalar 2003; 25:25-30. 
4. Herman CJ, Lengerich EJ, Stoodt G, Raleigh NC. Variation in recomendations for breast and cervical cancer screening among primary care physicians in North Caroline 1991. SM] [serial online] 1996 Jun. Disponível em: http://www.sma.org/smj/96jun6. htm. Acesso em: jun 2003.

5. Hakama M, Chamberlain J, Day, NE. Evaluation of screening programmes for gynaecological cancer. Br J Cancer 1985; 52:669-73.

6. Nyström L, Rutqvist LE, Wall S. Breast cancer screening with mammography: overview of Swedish randomised trials. Lancet 1993; 341:973-8.

7. Bolen JC, Rhodes L, Powell-Griner EE, Bland $\mathrm{SD}$, Holtzman D. State-specific prevalence of selected health behaviors, by race and ethnicity - behavioral risk factor surveillance system. 1997. [online] Disponível em: http:// www.cdc.gov/mmwr/preview/mmwrhtml/ ss4902al.htm. Acesso em: out 2002.

8. American Cancer Society. ACS Cancer Detection Guideline. 2003. Disponível em: http:// www. cancer.org/docroot/PEDcontent PED_2_3X_ACS_Cancer_Detection_ Guidelines_36.asp? sitearea $=$ PED. Acesso em: jan 2003.

9. Canadian Task Force. CTFPHC Sistematic reviews \& recommendations. $200 \mathrm{I}$. [online]. Disponível em: http://www.ctfphc.org. Acesso em: jan 2003.
10. Ashford A, Gemson D, Gorin SNS, Bloch S, Lantigua R, Ahsan H, Neugut Al. Cancer screening and prevention practices of inner-city physicians. Am J Prev Med 2000; 19:59-62.

II. Simó CE, Ureña TMM, Vernet VM, Sender PMJ, Larrossa SP, Jovell FE. Intervención del médico de familia en el diagnóstico del cáncer. Aten Primaria 2000; 26: 104-6.

12. Garcia-Giannoli H, Sasco AJ. Prévention des cancers par les médecins généralistes di Rhône: une enquête déclarative. Bull Cancer 1996; 83:853-63.

13. Bowman JA, Redman S, Reid ALA, SansonFisher RW. General practitioners and the provision of Papanicolaou smear-tests: current practice, knowledge and attitudes. Med J Aust 1990; 152: 178-83.

14. Grady KE, Lemkau JP, Mc Vay JM, Reisine ST. The importance of physicians encouragement in breast cancer screening of older women. Prev Med 1992; 21:766-80.

15. Sutton SM, Doner LD. Insights into the physician's role in mammography utilizations among older women. Womens Health Issues 1992; 2:175-9.

16. Battista RN, Palmer CS, Marchand BM, Spitzer WO. Patterns of preventive practice in New Brunswick. Can Med Assoc J 1985; 132:1013-5.
17. Gónzalez D, Maía E, Léon C, Alejandro M. Nódulo de mama: papel del médico general integral para su detección precoz mediante la enseñanza y el control del autoexamen de mama. Rev Cuba Med Gen Integr 1991; 7:328-34.

18. Miller AB, To T, Baines CJ, Wall C. Canadian National Breast Screening Study-2: 13-year results of a randomized trial in women aged 50-59 years. I Natl Cancer Inst 2000; 92:1490-9.

19. Tudiver F, Herbert C, Goel V. Why don't family physicians follow clinical practices guidelines for cancer screening?. CMAJ 1998; 159:797-8.

20. Santillán EA, Ponce EL, Peres M, Martínez ES, Castro JS, Ruíz PA. El conocimiento de profesionales de la salud sobre la prevención del cáncer cervical. Alternativas de educación médica. Salud Publica Mex 2000; 42:34-42.

21. Towler BP, Irwig L, Glasziou P, Weller D, Kewenter J. Screening for colorectal cancer using the faecal occult blood test, hemoccult. Cochrane Database Syst Rev 2000; (2): CD001216.

Artigo recebido: 30/09/2003 Aceito para publicação: 06/04/2004 